\title{
Main Chamber Sources and Edge Transport of Tungsten in H-mode Plasmas at ASDEX Upgrade
}

\author{
R. Dux, A. Janzer, T. Pütterich, and ASDEX Upgrade Team \\ Max-Planck-Institut für Plasmaphysik, EURATOM Association, D-85748 Garching \\ E-mail: Ralph.Dux@ipp.mpg.de
}

\begin{abstract}
In the fully tungsten clad ASDEX Upgrade, the sputtering rates of tungsten have been determined at all relevant plasma facing components using fast spectroscopic measurements with temporal resolution down to $0.5 \mathrm{~ms}$. The sputtering strongly increases during an edge-localised mode (ELM) and the ELMs are often the dominant cause for tungsten sputtering. A modelling approach was employed to calculate the tungsten source at the limiters and the resulting tungsten density at the pedestal top inside of the H-mode edge transport barrier (ETB). In the ETB, it is assumed that tungsten transport is collisional, i.e. behaves like other impurities. The collisional transport leads to strong inward drifts and steep density gradients in the ETB, which are flattened during an ELM causing an efflux of tungsten. The collisional transport in the ETB is also calculated for typical ITER conditions and the resulting tungsten density profiles as well as the transport of the helium ash through the ETB are evaluated.
\end{abstract}

PACS numbers: 52.55.Fa, 52.25.Fi, 52.25.Vy 


\section{Introduction}

The erosion rate of tungsten is much less than for the lighter elements, which substantially increases the lifetime of the respective plasma facing components (PFC) in a fusion reactor [1]. However, use of tungsten also implies the risk of strong central power losses by impurity radiation due to its high cooling factor at large temperatures. The influence of tungsten on the minimum triple product for ignition $n T \tau_{E}$ has been studied for a reference case with global He confinement time $\tau_{H e}^{*}=5 \tau_{E}$ and no other impurities [2]. It was found, that a central tungsten concentration of only $3 \times 10^{-5}$ increases the minimum triple product by $20 \%$ and at $1.9 \times 10^{-4}$ the ignition condition can not be satisfied. Due to this low concentration limit, a good understanding of the erosion mechanisms, the edge transport, and the radial transport in the confined plasma are mandatory to predict and control the tungsten source as well as the tungsten confinement. In the fully tungsten clad ASDEX Upgrade, these aspects can be studied. The central impurity transport and the control of central impurity accumulation has previously been investigated [3]. Here, we focus on the erosion, the penetration in the confined plasma and the transport in the edge transport barrier (ETB) of H-mode plasmas and investigate the relation between the erosion rates and the tungsten density at the pedestal top.

In H-mode plasmas with type-I ELMs, the tungsten dynamics at the edge is to a large extent governed by the ELMs [4]. The tungsten production at the plasma facing components is dominated by the erosion during ELMs mainly due to sputtering by light impurities. Nevertheless, discharges with a lower ELM frequency have a higher tungsten concentration in the confined plasma and a minimum ELM frequency is required to perform long steady discharges with constant tungsten concentration. The ELM frequency is usually increased by increasing the deuterium puff level. The flushing of tungsten from the confined plasma during the ELM seems to be dominant compared to the expected increase of tungsten production due to the same ELM [4].

It was found that the tungsten source at the outboard limiters has the strongest influence on the tungsten density in the confined plasma. For example, an increase of the limiter source obtained by an outward shift of the plasma column yields an increase of the tungsten density inside the separatrix by a factor of 3 . This density increase happens, even though the total tungsten sputtering rates in the outer divertor and at the heat shield are a lot larger. The sputtering rate at the outer divertor, essentially constant during the sweep, is $\approx 20$ times stronger, while the tungsten source rate at the inner heat shield is $\approx 2$ times higher and decreases during the outboard shift [4]. The minor role of the tungsten source in the outer divertor is also consistent with experiments and modelling on the divertor retention of tungsten [5, 6, 7]. Even though further experiments are needed to better quantify the divertor retention for various operation scenarios, a simplifying assumption used in this study is to neglect the divertor sources. Thus, a modelling approach with an upgraded version of the $1 \mathrm{D}$ radial impurity transport code STRAHL was employed to calculate the erosion, 
penetration and edge transport of tungsten eroded at the limiters. Using this approach, the calculated confinement times of tungsten form an upper bound since only the limiter source is assumed to be responsible for the measured tungsten concentration in the confined plasma.

One important modelling element is the radial impurity transport in the ETB. Here, the density profile evolution of helium, carbon, neon and argon was measured with charge exchange recombination spectroscopy [8] and it was found that between ELMs, all impurities are subject to a strong inward pinch leading to steep impurity density gradients in the ETB which are flattened during an ELM. The evaluated impurity transport coefficients between ELMs are in accordance with neo-classical theory and cause an increase of the peaking with rising impurity charge state $[9,8]$. Thus, we assume also predominantly neo-classical transport for tungsten and find an even stronger peaking than for the light elements. The effect of the ELM is modelled by a strong increase of the diffusion coefficient. This flattens the steep negative gradient of the impurity density in the ETB and leads to an efflux of impurities from the confined plasma. Since the code models tungsten and light impurities in one run, the efflux of light impurities onto the limiters can be used to self-consistently model the time history of tungsten erosion by physical sputtering and the radial transport of tungsten in the plasma edge.

New measurements of ELM resolved erosion rates, which complement previous local measurements, will be discussed in section 2. The main elements of the transport model are described in section 3. The model is not predictive and contains parameters which are not well known. Therefore, the dependence of the modelled tungsten confinement on the radial and parallel transport parameters and the ELM frequency are discussed in section 4. In section 5, the transport model is applied to fit experimental values from ASDEX Upgrade H-mode discharges with different ELM frequencies. The study described there evaluates the relative probability of extracted transport parameters from the knowledge of the tungsten density at $r / a \approx 0.8$ for a given tungsten erosion rate at the outboard limiters. In ITER, temperatures in the ETB will be much higher than in ASDEX Upgrade and the neo-classical impurity transport coefficients are more than an order of magnitude smaller. We discuss in section 6 the consequences of a prevailing neo-classical impurity transport in the ETB on the edge profiles of tungsten and on the transport of the helium ash through the ETB.

\section{ELM Resolved Tungsten Erosion Rates}

The experimental information on the influx of tungsten and on the $\mathrm{W}$ density in the confined area is gained from spectroscopic measurements. Tungsten influx is measured in the visible spectral range using a WI spectral line at $400.9 \mathrm{~nm}$. Simultaneous measurements on 38 lines-of-sight are used to quantify the influx at all major erosion areas, i.e. the outer divertor, the heat shield at the inner column and the outboard limiters (a sketch of the PFC geometry and the lines-of-sight is shown in [4]). The W 
concentration $c_{W}$ in the outer part of the confined plasma is deduced from W-spectra in the VUV region. The quasi-continuum at $5 \mathrm{~nm}$ emitted by ions around $\mathrm{W}^{30+}$ yields $c_{W}$ at $T_{e} \approx 1.5 \mathrm{keV}$ [10], i.e. well within the ETB typically at $r / a=0.8$ for the H-mode plasmas discussed below. Another quasi-continuous spectral feature around $15 \mathrm{~nm}$ is presently studied to quantify the concentration of lower ion stages around $\mathrm{W}^{20+}$ [11].

The effect of ELMs on the tungsten influx from the main chamber plasma facing components (PFC) has previously been determined by fast spectroscopic measurements on a single line-of-sight observing one spot in the middle of one low-field side limiter [12] or in the divertor [4]. The repetition time of the measurement was $253 \mu \mathrm{s}$. The total erosion rates can only be estimated, when the poloidal $\mathrm{W}$ influx profiles along the PFC are available, which requires measurements on many lines-of-sight for each PFC [4]. However, simultaneous recording of about 10 spectra increases the minimum repetition time of the CCD-cameras to about $4 \mathrm{~ms}$, which is often too slow to have enough data points in the measured time series that are not affected by ELMs. When adding the spectra of all lines-of-sight on the camera into one single binned spectrum (the charges which are created in all pixels at one wavelength are combined during read out), the repetition time can be reduced substantially to $0.5 \mathrm{~ms}$ for the cameras measuring the limiters and the outer divertor and to $1.2 \mathrm{~ms}$ for the camera recording the spectra from the inner column. As will be explained later in this section, the calculation of the total influx rate from the binned spectra can be performed, when knowing the respective weights of each individual line-of-sight in the binned spectrum, which depend on the influx profiles.

Thus, the investigated discharges were performed twice, with slower profile measurements in the first pulse and with faster recording using spectra binning in the second discharge. For the slow measurements, the total influx rate $\Phi$ in [atoms/s] is calculated by first fitting the WI line of each spectrum, which yields a count rate $\dot{N}_{i}$ emitted by the WI line on the $i$ th line-of-sight. $\dot{N}_{i}$ is multiplied with the sensitivity $S_{i}$ to get a line radiance in [photons $\left./\left(\mathrm{m}^{2} \mathrm{~s} \mathrm{sr}\right)\right]$. Multiplying further the line radiance with $4 \pi S /(X B)$ delivers the local influx density, where the number of ionisations per emitted photon $S /(X B)=20[13,7,14]$ was used. This is multiplied with an effective area $A_{i}$ and finally, the sum is taken over all lines-of-sight:

$$
\Phi=4 \pi \frac{S}{X B} \sum_{i} A_{i} S_{i} \dot{N}_{i}
$$

The sum of the count rates equals the count rate of the binned spectrum $\dot{N}_{b}=\sum_{i} \dot{N}_{i}$ and yields the conversion factor $k_{\Phi}=\Phi / \dot{N}_{b}$ for the calculation of the rate from the binned data. This factor $k_{\Phi}$ depends on the shape of the plasma with respect to the shape of the PFC, i.e. where the PFC is mainly hit, and is slowly varying in time during the flat top phase of the investigated discharges.

Fig.1 shows an example for the evolution of the tungsten source from the outboard limiters during a type-I ELM for pulse \#25095, a discharge of the series discussed in the next paragraph. The data were recorded with an exposure time of $0.5 \mathrm{~ms}$ and cover 117 ELMs during a 1s time interval. All measurements are mapped on a common time base 


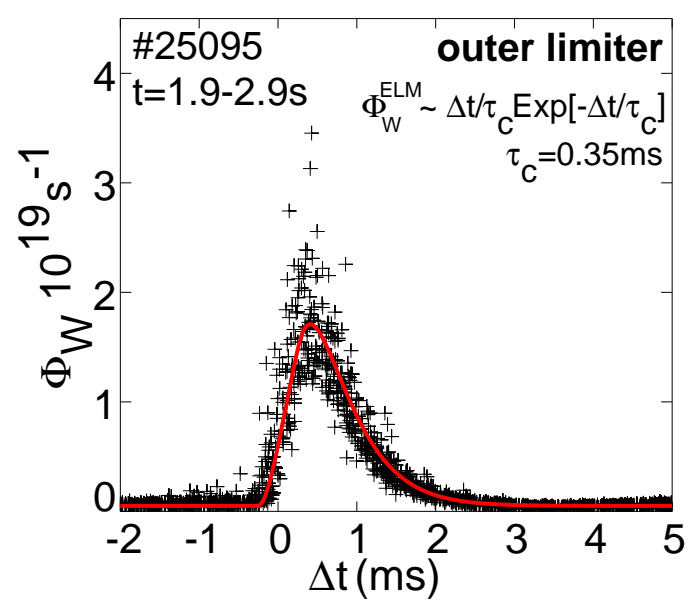

Figure 1. Evolution of the W source rate $\Phi_{W}$ at the outboard limiters during a type-I ELM. The temporal co-ordinate $\Delta t$ is the time difference to the arrival time of the ELM in the outboard divertor and the data of 117 ELMs are overlaid. The individual data point averages over an exposure time $\Delta t_{\text {exp }}=0.5 \mathrm{~ms}$.

$\Delta t=t-t_{E L M}$ giving the time difference to the arrival time $t_{E L M}$ of the ELM in the outboard divertor. The red curve shows a fit to the data using a simple model function for the source rate:

$$
\Phi_{W}(\Delta t)=\Phi_{W, o f f}+\left(\Phi_{W, \max }-\Phi_{W, o f f}\right) \frac{\Delta t}{\tau_{c}} \exp \left[1-\frac{\Delta t}{\tau_{c}}\right] \Theta(\Delta t)
$$

Here, $\Phi_{W, o f f}$ is the rate between ELMs, $\Phi_{W, \max }$ is the maximum rate during the ELM, $\tau_{c}$ is a characteristic time and $\Theta(\Delta t)$ is the Heaviside function. The parameters for the example of Fig. 1 are $\Phi_{W, o f f}=5 \times 10^{17} \mathrm{~s}^{-1}, \Phi_{W, \max }=1.5 \times 10^{19} \mathrm{~s}^{-1}$ and $\tau_{c}=0.35 \mathrm{~ms}$. The function is convolved with a boxcar function of $0.5 \mathrm{~ms}$ width to obtain the red curve in the figure. The time duration with increased source due to an ELM $\Delta t_{E L M}$ is about 2 ms. Outside of this interval the ELM peak has dropped below $5 \%$ of its maximum value.

A series of type-I ELMy H-mode discharges with $I_{p}=1 \mathrm{MA}, B_{T}=2.5 \mathrm{~T}, q_{95}=4.5$, $P_{N B I}=7.5 \mathrm{MW}, P_{E C R H}=1.6 \mathrm{MW}$ and $\bar{n}_{e}=7.5 \times 10^{19} \mathrm{~m}^{-3}$ were performed, which had slow radial shifts of the plasma column, such that either the inner column or the outboard limiters were closer to the separatrix. We designate flux surfaces by $\Delta R$, the radial distance of the flux surface to the separatrix on the low-field side at the height of the magnetic axis and define the distance of a main chamber PFC to the separatrix by the lowest value of $\Delta R$ on the poloidal shape of that component, again, referenced to the outer midplane. For the limiters, the shifts covered a range $\Delta R_{\text {lim }}=4.2-7.2 \mathrm{~cm}$ and for the heat shield $\Delta R_{H S}=3.2-5.5 \mathrm{~cm}$. The ELM frequency $f_{E L M}$ was varied by applying different deuterium puff levels and covers a range $f_{E L M}=30-130 \mathrm{~Hz}$. The average energy loss per ELM was in the range $\Delta W_{E L M}=19-50 \mathrm{~kJ}$.

A database was formed by dividing the evolution of the tungsten erosion rate during the radial sweeps into intervals of $100 \mathrm{~ms}$ duration and forming mean values for each 


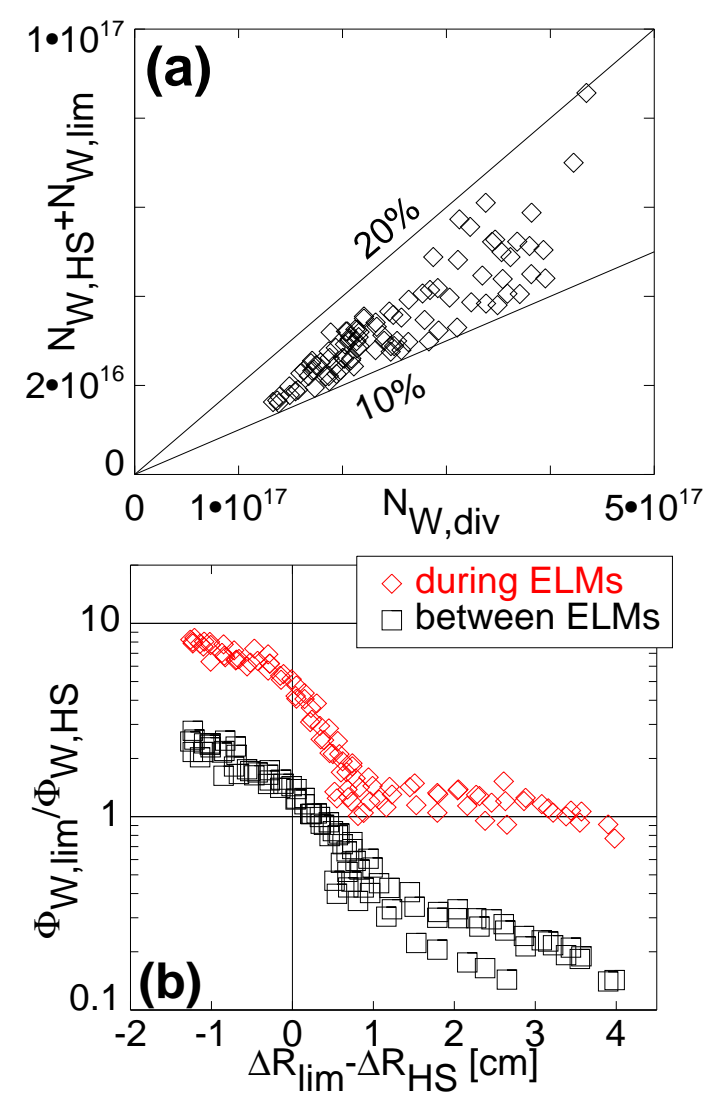

Figure 2. (a): ELM erosion at the main chamber components versus erosion at the outer divertor. (b): Ratio of the erosion rates of inner heat shield to the outboard limiters versus the difference of the distances of the respective component to the separatrix.

interval. An ELM causes a large spike of the influx rate and for each interval, the offset rate $\Phi_{\text {off }}$ between ELMs was evaluated. To this end, we calculate the fraction $f_{\text {off }}$ of data points which are not affected by ELMs: $f_{\text {off }}=\left(\Delta t_{\text {cycle }}-\Delta t_{E L M}-\Delta t_{\text {exp }}\right) / \Delta t_{\text {cycle }}$. Here, $\Delta t_{\text {cycle }}=1 / f_{E L M}$ is the average duration of the ELM cycle, $\Delta t_{E L M}=2 \mathrm{~ms}$ is the duration of increased influx due to an ELM (see discussion of fig.1) and $\Delta t_{\text {exp }}=0.5,1.2 \mathrm{~ms}$ is the exposure time of the camera. Sorting the $n$ measured rates in ascending order, the first $n f_{\text {off }}$ data points are offset values and the mean of these values was taken for $\Phi_{\text {off }}$. The difference to the mean value $\langle\Phi\rangle$ of the $100 \mathrm{~ms}$ time interval represents the mean rate during ELMs.

$$
N_{W}=\frac{\langle\Phi\rangle-\Phi_{o f f}}{f_{E L M}}
$$

is the mean number of eroded tungsten atoms per ELM, and

$$
c_{E L M}=\frac{\langle\Phi\rangle-\Phi_{o f f}}{\langle\Phi\rangle}
$$

is the fraction of the total sputtering rate, which is caused by ELMs. 
Fig.2(a) shows the number of eroded tungsten atoms per ELM for the main chamber components $N_{W, \text { lim }}+N_{W, H S}$ versus the eroded atoms in the divertor $N_{W, \text { div }}$. In the main chamber, an ELM causes an erosion between (10-20)\% of the divertor value. The ELM erosion dominates the sputtering at the limiters. All values for the ELM fraction at the limiters $c_{E L M, l i m}$ are in a range 0.6-0.8 with no trend on the energy loss per ELM or the ELM frequency, i.e. larger ELMs, which come with lower ELM frequency, contribute as much as faster, lower energy ELMs. There is a trend with the distance from the limiter to separatrix $\Delta R_{\text {lim }}$. The values of $c_{E L M, l i m}$ are around 0.65 for $\Delta R_{\text {lim }} \approx 4.5 \mathrm{~cm}$ and rise to 0.8 for $\Delta R_{\text {lim }} \approx 6.5 \mathrm{~cm}$. Thus, the ratio of ELM to inter-ELM W limiter sources rises with $\Delta R_{\text {lim }}$ by about a factor of 2 and the decay length is by the same factor longer during an ELM than between ELMs. For the ELM fraction at the heat shield $c_{E L M, H S}$, the values are in a range 0.3-0.5 without a clear dependence on ELM energy or distance to the separatrix. These results agree with the earlier local measurements at the limiters [4], however, at the heat shield we now find $c_{E L M, H S}$ values which are lower by 0.2 than before, where the present spectra have much better data quality, especially between ELMs. In Fig.2(b) rates at the limiters and at the heat shield are compared. It shows the ratio of the respective sputtering rates versus the difference of the distances to the separatrix. Positive values of the x-coordinate $\left(\Delta R_{\text {lim }}-\Delta R_{H S}\right)$ represent plasmas, where the outboard limiters are further away from the separatrix than the heat shield. For the flux between ELMs, $\Phi_{W, \text { lim }} / \Phi_{W, H S}$ approximately passes through 1 for about equal distance to the separatrix given the systematic uncertainties of the equilibrium reconstruction of $\approx 0.5-1 \mathrm{~cm}$ and the flux measurements (factor $\approx 2-3$ ). For large limiter distances, the source ratio deacays with reduced slope. Thus, the inter-ELM tungsten sources at the inboard and outboard components decrease with the same decay length for lower $\Delta R$ values. The data points with reduced slope belong to cases with very large $\Delta R_{\text {lim }}$ above $5.5 \mathrm{~cm}$, where the source at the limiter decreases with a large decay length (very large distances of the heat shield are not in the data set). During ELMs, however, substantially more influx comes from the outboard limiters and the ratio increases by a factor of 4-5. Thus, the ion flux and/or the temperatures, which strongly determine the erosion yield, increase more at outboard components than at the inner column during an ELM.

\section{Impurity Transport Model}

Modelling of the impurity transport in principle requires a three dimensional code which includes the impurity production at the limiters and the transport perpendicular and parallel to the magnetic field lines. 3D or 2D codes are computationally very demanding and here, a first approach is done with a 1D impurity transport code.

The STRAHL code [15] solves the coupled radial continuity equations for the flux surface averaged densities $n_{z}$ of each ionised stage of an impurity. The code can model light impurities and tungsten during one run in order get a more realistic description of collisional radial tungsten transport and tungsten erosion during the ELM 
cycle. For the ASDEX Upgrade runs, carbon and oxygen were included, while for the ITER simulations, helium, oxygen and argon were considered. The background plasma parameters like $n_{e}, T_{e}$ and $T_{i}$ are input parameters to the code, and the main ion density is calculated from the quasi-neutrality condition. For the radial transport, turbulent diffusion coefficients and drift velocities are set ad hoc (for historical reasons, turbulent transport is also termed anomalous transport). Usually, the anomalous coefficients are set to fit the measured evolution of the impurity density profiles after a fast disturbance [16]. Here, we will study the effect of different settings for the turbulent transport in the edge of the plasma on the confinement of tungsten (see below). The neo-classical transport parameters are calculated using the NEOART code [17, 15], which has been included as a subroutine to STRAHL.

The radial co-ordinate $r$ used in STRAHL is defined by the volume $V$ enclosed by the flux surface $r=\sqrt{V /\left(2 \pi^{2} R_{a x i s}\right)}$. For an impurity transport code it is essential, that the calculation domain extends into the scrape-off layer (SOL), where the impurity sources are located. However, in a diverted plasma $r$ and $V$ are not defined in the SOL and the volume change per poloidal flux change $d V / d \Psi$ diverges when approaching the separatrix. The commonly adopted workaround is to blow-up the last closed flux surface to match for a given poloidal flux value the true flux surface at the height of the magnetic axis. When comparing the major radius at the low-field side equator $R_{l f s}$ with $r$, we have for the investigated ASDEX Upgrade discharges $d r / d R_{l f s} \approx 1.8$ around the separatrix and in the SOL, i.e. a radial gradient in the ETB appears in the co-ordinate $r$ a factor of $\approx 1.8$ less steep than the measured gradient at the low-field side equator.

Outside the separatrix, the parallel transport towards the divertor or to the limiting elements in the main chamber is simply described by volumetric losses, i.e. by adding a term $-n_{i m p} \nu_{\|}$to the transport equation being proportional to the parallel loss frequency $\nu_{\|}$. A sketch of the parallel losses and the tungsten influx in STRAHL is shown in figure 3. The loss frequency $\nu_{\|}$is given by the parallel connection length between the surface elements, $L_{\|}$, and the mean flow velocity of the impurities towards the surfaces, $v_{\|, i m p}$, i.e.

$$
\nu_{\|}=\frac{2 v_{\|, i m p}}{L_{\|}}
$$

The SOL is divided into a divertor SOL with a large connection length of $50 \mathrm{~m}$ and a limiter SOL with $1 \mathrm{~m}$ connection length. Thus, $\nu_{\|}$strongly increases when entering the limiter SOL. The mean flow velocity of the impurities, $v_{\|, i m p}$, is poorly known and essentially a free input parameter to the code. It will be used later to fit the measured tungsten confinement. In the divertor SOL, the collision frequency of the impurities is much larger than the loss frequency, and the impurities are entrained in the deuterium flow

$$
v_{\|, i m p} \approx v_{\|, D}=M \sqrt{\frac{k_{B}\left(3 T_{i}+T_{e}\right)}{m_{D}}},
$$

which is described by the adiabatic acoustic speed and an average Mach number $M$. The Mach number $M$ is set ad hoc and assumed to be constant throughout the entire 


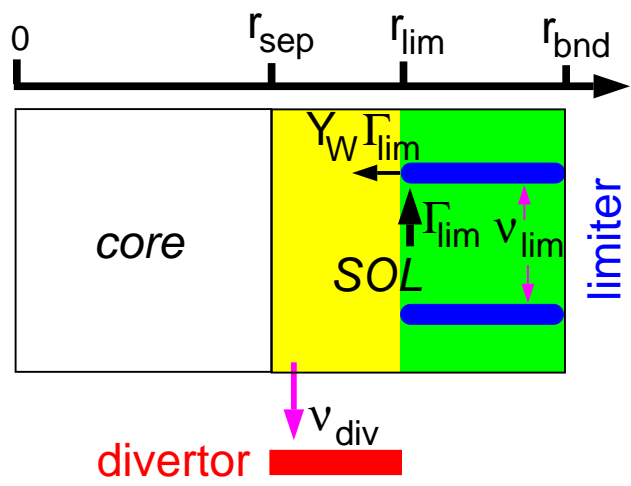

Figure 3. Sketch of the parallel losses and the tungsten influx in STRAHL.

SOL. This description allows to change $v_{\|, i m p}$ for all impurities by changing just one number in the input parameter set discounting details in the radial shape of $v_{\|, i m p}$ that can anyway not be resolved by our measurements. In the limiter SOL, the densities are very low, the parallel heat conduction is always sheath limited and only small parallel temperature gradients are expected [18]. Furthermore, the impurity collisionality is small, i.e. there is only a weak coupling of deuterium and impurity flow speed, and kinetic effects are important. Here, the isothermal acoustic speed of the impurity $v_{\|, i m p}=\sqrt{k_{B}\left(T_{i}+T_{e}\right) / m_{i m p}}$ would be more appropriate. For $\mathrm{W}$ at $T_{i}=2 T_{e}$, this formula yields the same result as eq. 6 when using $M=0.068$, which is within a factor of 2 the Mach number that was needed to fit the ASDEX Upgrade results. Having these complications in mind, the loss frequency in the whole SOL is calculated with eq. 5 and 6 , expecting the large difference in the connection length to be the prevailing effect when entering the limiter shadow. The radial grid extends up to the boundary radius $r_{b n d}$. Here, a boundary condition $d n_{z} / d r=-n_{z} / \lambda$ with radial decay length $\lambda=\sqrt{D\left(r_{b n d}\right) / \nu_{\|}\left(r_{b n d}\right)}$ is used.

The neutral impurities start at the limiter tip with $r=r_{\text {lim }}$, which is a simplification of the radial distribution of starting positions. Furthermore, they have a constant radial velocity $v_{0}$. Their density decays radially according to the rate $\nu_{i o n}=n_{e}\left\langle\sigma_{\text {ion }} v_{e}\right\rangle$ for ionisation:

$$
n_{0}(r)=n_{0}\left(r_{l i m}\right) \frac{r_{l i m}}{r} \exp \left[-\int_{r_{l i m}}^{r} \frac{\nu_{i o n} d r}{v_{0}}\right] .
$$

For the light impurities, the neutral impurity influx rate is set ad hoc to get the desired impurity concentration in the confined plasma. Complete recycling at the limiters can be included by adding the loss rates onto the limiters to the influx. For tungsten, the code calculates the source rate of $\mathrm{W}$ using erosion yields for physical sputtering. To this end, the rate of ions with charge $Z$ that are lost to the limiters is determined using:

$$
\Phi_{\|, \text {lim }}=2 \pi R_{0} \int_{r_{\text {lim }}}^{r_{\text {bound }}} \nu_{\|}(r) n_{z}(r) 2 \pi r d r
$$

This rate is multiplied with the sputtering yield $Y_{W}(E, m)$ and the sum over all charge stages of all elements yields the total influx rate. The yield depends on the energy and 
the mass of the impinging ions. The energy of the impinging ions is approximated by the value for a collision-less deuterium plasma [19], which yields

$$
E=2 k_{B} T_{i}+3 Z k_{B} T_{e}
$$

for $T_{i}=2 T_{e}$. The first term is the average ion energy at the sheath entrance and the second term is due to acceleration within the sheath. The yield is calculated with the revised Bodhansky formula as given in [20]. The energy distribution of the sputtered W atoms is assumed to be a Thompson distribution [21] with a cos-angular distribution. It depends on the mass and the energy of the sputtering ion, where the energy is a function of the ion charge $Z$ for a given $T_{e}$ via eq.9. For each sputtering element and ion stage, the code calculates the mean radial starting velocity $v_{0}$ of $\mathrm{W}$ from the distribution. For tungsten, also the fraction of promptly redeposited ions is calculated. Prompt redeposition, i.e. the immediate return to the surface during the first gyration after ionisation, depends on the product of cyclotron frequency of singly ionised tungsten $\omega_{c}=e B / m_{W}$ and the time delay of the ionisation after the impurity starts at the surface, $\Delta t_{i o n}$. For a homogeneous plasma and a cosine angular distribution of the starting velocities, the fraction of particles that do promptly redeposit is:

$$
f_{\text {redep }}=\frac{1}{1+\left(\Delta t_{\text {ion }} \omega_{c}\right)^{2}}
$$

The code uses this simple prescription to estimate the prompt redeposition at each radial grid point, where $\Delta t_{i o n}=\left(r_{l i m}-r\right) / v_{0}$ is used. The redeposited particles are removed from the radial profile of the neutrals, i.e. eq. 7 is multiplied with $\left(1-f_{\text {redep }}(r)\right)$ such that the effective volume source of ions at radius $r$ becomes $n_{0}(r)\left(1-f_{\text {redep }}(r)\right) \nu_{\text {ion }}(r)$. For each fractional $\mathrm{W}$ source rate due to sputtering by one ion species, STRAHL calculates the radial profiles of the neutral $\mathrm{W}$ as described above and removes from this contribution the prompt redeposition profile. Finally, it adds up the neutral density profiles for sputtering by all species. For helium in ITER, also the volume source due to fusion was included.

For the modelling of the impurity behaviour in the edge plasma, radial transport coefficients in the edge, roughly for $r / a>0.7$, are needed. From previous experimental impurity transport studies in ASDEX Upgrade H-mode plasmas, there is good knowledge about the radial transport coefficients inside of the ETB and in the ETB, while transport coefficients in the SOL are not known. In the edge of the core plasma but still on the inside of the ETB, impurity transport is dominated by plasma turbulence and diffusion coefficients have typical values of a few $\mathrm{m}^{2} \mathrm{~s}^{-1}$, while $v / D$ values are low. This was shown by investigations on helium, neon [22], silicon [23] and argon [24]. Further out in the ETB, recent impurity transport investigations on helium, carbon, neon and argon have shown, that the radial transport in the ETB between ELMs is in accordance with collisional transport coefficients $[9,8]$, i.e. in the ETB turbulent impurity transport is low with respect to the collisional level. All impurities are in the Pfirsch-Schlüter regime and the collisional transport coefficients are just the sum of the classical (CL) and the Pfirsch-Schlüter (PS) contribution. Thus, the collisional diffusion coefficient $D_{\text {neo }}$ is 


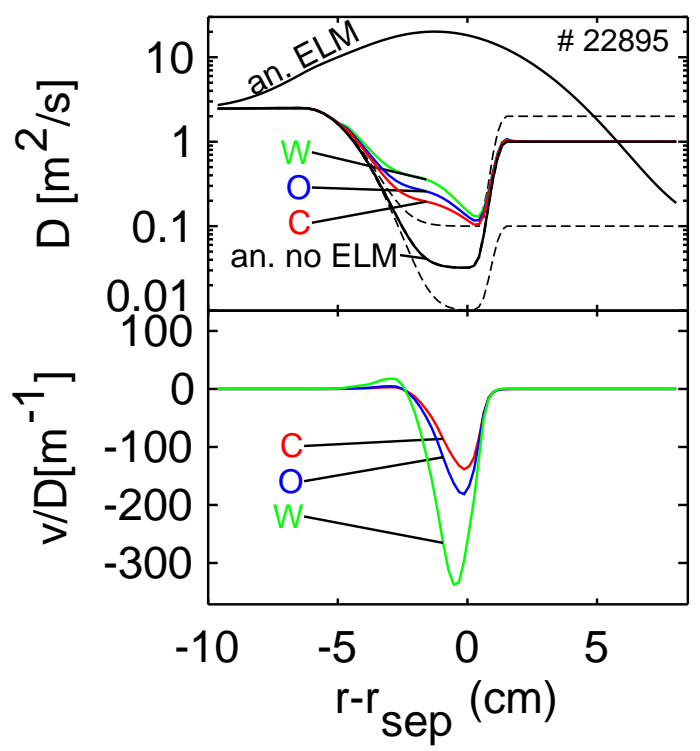

Figure 4. Profiles of impurity diffusion coefficient (upper box) and drift parameter (lower box) for the modelling of AUG discharge \#22895. In the upper box, the lower solid black line (an. no ELM) is the assumed turbulent diffusion coefficient between ELMs $D_{a n}^{n o E L M}$. The coloured lines are the sum of neoclassical diffusion coefficients $D_{n e o}$ and $D_{a n}^{n o E L M}$ for the different elements, and the upper solid black line (an. ELM) is the diffusion coefficient at the start of the ELM. It decays linearly within $1 \mathrm{~ms}$ to the lower black line. The black dashed lines indicate the boundaries of the $D_{a n}^{\text {no } E L M}$ profile, that were covered in the sensitivity analysis. In the lower box, the different lines represent $v / D=v_{n e o} /\left(D_{n e o}+D_{a n}^{n o} E L M\right)$ for the quiet phase between ELMs. During the $\operatorname{ELM} v / D$ is negligible.

$D_{\text {neo }}=D_{C L}+D_{P S}$ and the collisional radial drift velocity $v_{\text {neo }}$ is $v_{\text {neo }}=v_{C L}+v_{P S}$. The collisional radial transport is characterised by a strong inwardly directed pinch velocity $v_{n e o}$ and the ratio $v_{n e o} / D_{\text {neo }}$ increases with the impurity charge. Tungsten has even higher collisional transport coefficients than the light impurities and its transport must be dominated by the effect of Coulomb collisions. The ratio $v_{n e o} / D_{\text {neo }}$ is expected to be larger due to the higher charge. The collisional transport of tungsten is dominated by collisions with low-Z impurities and it is necessary to include impurity-impurity collisions in the calculation of the transport coefficients of W. With increasing low-Z impurity concentration the diffusion coefficient $D_{\text {neo }}$ increases. The impurity diffusion coefficient in the SOL is not well known, however, it will be shown below, that the fit of the discussed measurements by the model is very insensitive to this parameter.

The elements of the radial transport model are depicted in Fig.4. The turbulent transport coefficients $D_{a n}$ and $v_{a n}$ are assumed to be equal for all charge stages of all impurities. In the phase between ELMs, the anomalous diffusion coefficient was reduced in the ETB to $D_{a n}^{E T B}$ (solid black line) being well below the collisional diffusion coefficient of all impurities. In Fig.4 it is about a factor of 10 below the collisional values where $\mathrm{W}$ has the largest diffusion coefficient. The curves in colour show the sum 
of anomalous and collisional diffusion coefficients for each element. The total values of the drift parameter $v / D$ in this phase are shown in the lower box. The turbulent drift velocity is assumed to be negligible compared to the neoclassical drift and is set zero. Thus, the drift parameter is $v / D=v_{\text {neo }} /\left(D_{\text {neo }}+D_{a n}\right)$. W has the strongest inwardly directed drift parameter. The minimum is just inside the separatrix. For $r \geq r_{\text {sep }}$, the neoclassical coefficients are not defined, however, for the numerical solution of the transport equations, a large discontinuity in the transport coefficients at the separatrix must be avoided. Therefore, the logarithmic divergence of the safety factor was circumvented by linearly extrapolating the radial dependence of $q$ between $\rho_{\text {pol }}=0.9$ and 0.95 up to the separatrix. Thus, the collisional transport coefficients were calculated up to $r=r_{\text {sep }}+1.5 \mathrm{~cm}$ and switched off for larger radii. From $r=r_{\text {sep }}+0.5 \mathrm{~cm}$ to $r=r_{\text {sep }}+1.5 \mathrm{~cm}$, the anomalous diffusion coefficient in the inter-ELM phase is increased to a second plateau $D_{S O L}$. During a sensitivity scan (see below), $D_{S O L}$ and $D_{a n}^{E T B}$ were independently varied by a factor of 10 and the boundaries of the corresponding $D_{a n}$ profiles are indicated by the dashed lines. An ELM is induced by a sudden switch-on of a large diffusion coefficient in the edge (upper black curve), which decays linearly in time within $1 \mathrm{~ms}$.

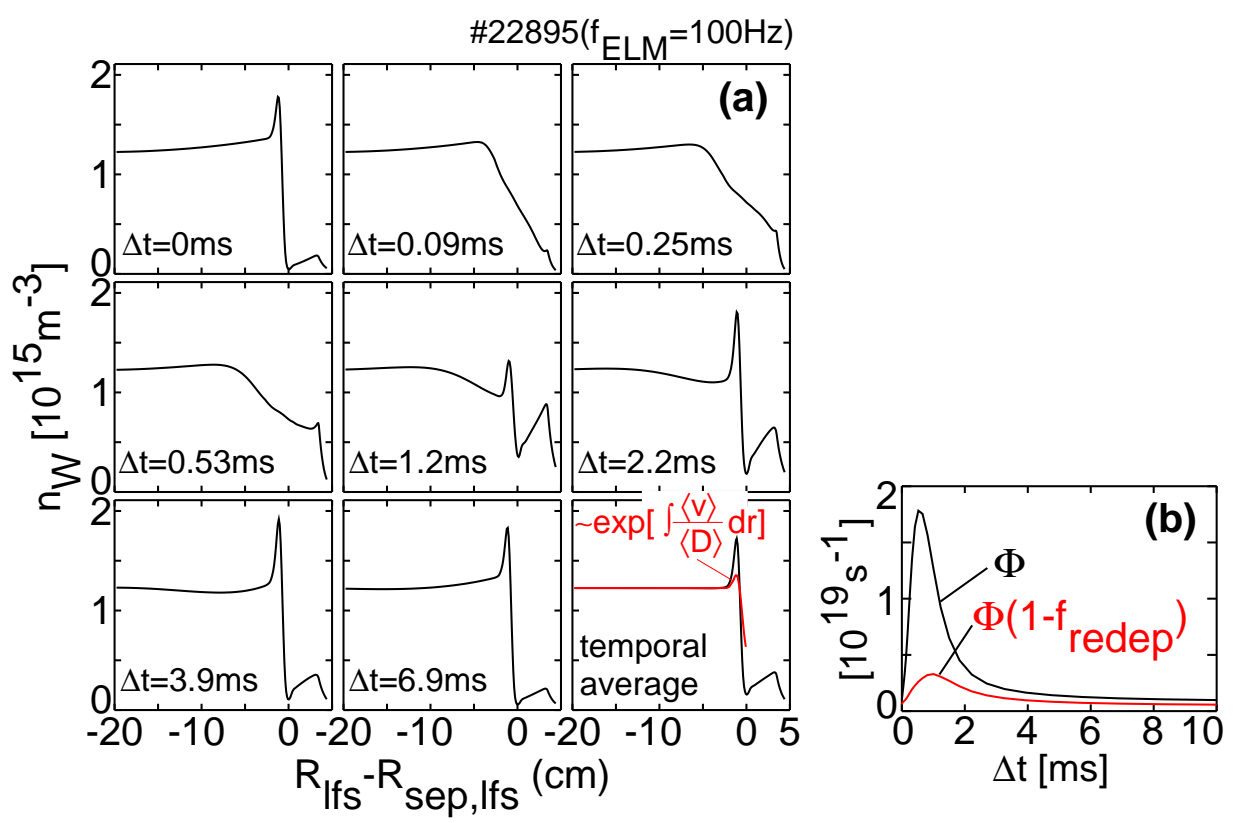

Figure 5. In fig.(a), the modelled evolution of the total tungsten density $n_{W}$ during an ELM cycle of $10 \mathrm{~ms}$ duration is shown for AUG discharge \#22895. $D_{S O L}=1 \mathrm{~m}^{2} \mathrm{~s}^{-1}$ and $D_{a n}^{E T B}=0.032 \mathrm{~m}^{2} \mathrm{~s}^{-1}$ was used. The lower right frame shows the temporally averaged profile in comparison with the profile of $\exp \left[\int(\langle v\rangle /\langle D\rangle) d r\right]$. Fig.(b) depicts the time evolution of the tungsten sputtering rate $\Phi$ and effective source $\Phi\left(1-f_{\text {redep }}\right)$.

An example of the modelled density evolution of the total tungsten density $n_{W}$ during an ELM cycle of $\Delta t_{E L M}=10 \mathrm{~ms}$ duration for discharge \#22895 is shown in Fig.5(a). Here, the settings for the two plateaus of the turbulent diffusion coefficient 
were $D_{S O L}=1 \mathrm{~m}^{2} \mathrm{~s}^{-1}$ and $D_{a n}^{E T B}=0.032 \mathrm{~m}^{2} \mathrm{~s}^{-1}$ (other parameters are in Tab.1). The radial co-ordinate is the distance to the separatrix at the low-field side equator. All impurities are in quasi-equilibrium, i.e. the temporal average density over an ELM cycle

$$
\langle n\rangle=\frac{1}{\Delta t_{E L M}} \int_{0}^{\Delta t_{E L M}} n(t) d t
$$

is constant in time. The profile of $\left\langle n_{w}\right\rangle$ is depicted in the lower right box and the other boxes show various time points $\Delta t$ with respect to the start of the ELM. The gradients of the strongly peaked edge profile at $\Delta t=0 \mathrm{~ms}$ are flattened in the first few $100 \mu \mathrm{s}$ after the start of the ELM. At the same time the tungsten sputtering rate $\Phi$ rises as is shown in Fig.5(b), however, the prompt redeposition leads to a much smaller increase of the effective source $\Phi\left(1-f_{\text {redep }}\right)$. After $\Delta t=1 \mathrm{~ms}$, the edge gradient recovers till the start of the next ELM. At a major radius of $\approx-1.2 \mathrm{~cm}$ inside of the separatrix, a peak of the density evolves, which is due to a maximum in the $v / D$ profile at slightly positive (outward directed) value of $17 \mathrm{~m}^{-1}$. Inside of $-2.5 \mathrm{~cm}$, the drift velocity is zero. Nevertheless, there is still a slightly hollow profile up to about $-20 \mathrm{~cm}$ for about the second half of the ELM cycle. However, the average profile is perfectly flat in that region and has just the expected shape, since inside the separatrix, the tungsten source is zero and the temporally averaged radial flux density $\left\langle\Gamma_{w}\right\rangle$ must be zero as well: $\left\langle\Gamma_{w}\right\rangle=-\left\langle D d n_{w} / d r\right\rangle+\left\langle v n_{w}\right\rangle=0$. For a weak modulation, the following linearization can be done.

$$
1=\frac{\left\langle v n_{w}\right\rangle}{\left\langle D d n_{w} / d r\right\rangle}=\frac{\langle v\rangle\left\langle n_{w}\right\rangle}{\langle D\rangle d\left\langle n_{w}\right\rangle / d r} \quad \rightarrow \quad \frac{d\left\langle n_{w}\right\rangle / d r}{\left\langle n_{w}\right\rangle}=\frac{\langle v\rangle}{\langle D\rangle}
$$

Inside of $\approx-2 \mathrm{~cm}$, the modulation is sufficiently weak and the average profile can just be calculated from the time averaged transport coefficients. Inside of $-2.5 \mathrm{~cm}, v$ is anyway zero for all times and $\left\langle n_{W}\right\rangle$ is flat. Closer to the separatrix, the modulation is strong and the averaged gradients are steeper than in the linearised approximation.

\section{Main Dependences of the Tungsten Transport Model}

The model was applied to three discharge phases with different deuterium puff levels $\Phi_{D}$ and ELM frequencies (see Tab.1). The model has previously been applied to these discharges [25] in order to find one consistent solution, without studying the dependence on the above mentioned parameters. Another difference with respect to [25] is, that on top of the turbulent $D_{S O L}$, neoclassical transport coefficients were used within the divertor SOL, which has been avoided in this work.

Before trying to fit the measurements of individual discharges with the model, it is important to study and understand the dependence of the modelling results on the free knobs of the code, i.e. the parameters, which are not well known from theory or measurement. In our case, these free parameters are the perpendicular and parallel transport parameters in the SOL. To this end, an independent scan of the ELM frequency with $f_{E L M}=25,50,100$, and $200 \mathrm{~Hz}$, the inter-ELM anomalous diffusion coefficients $D_{\text {an }}^{E T B}=0.01,0.0316$ and $0.1 \mathrm{~m}^{2} \mathrm{~s}^{-1}, D_{S O L}=0.1,0.316,1$ and $2 \mathrm{~m}^{2} \mathrm{~s}^{-1}$ and 


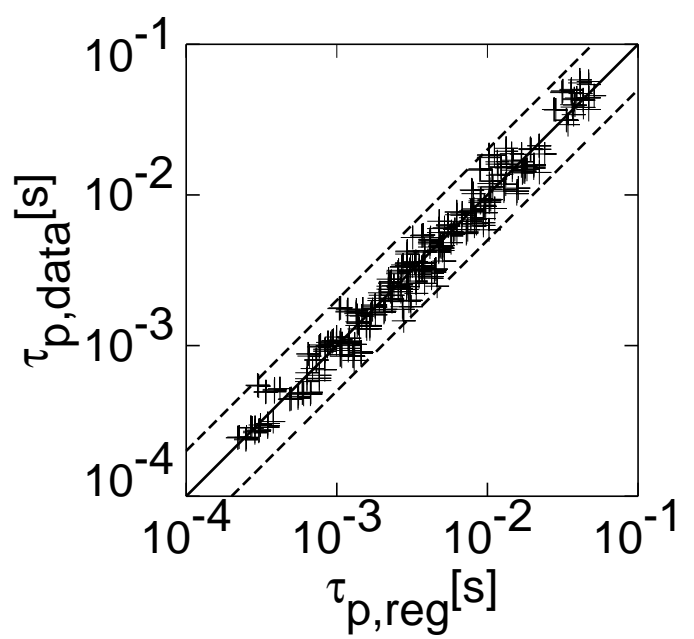

Figure 6. Regression of the particle confinement time of tungsten $\tau_{p}$ on the ELM frequency, the average parallel loss time from SOL to divertor $\left\langle\left\langle\tau_{S O L \rightarrow \text { div }}\right\rangle\right\rangle$ and the SOL diffusion coefficient $D_{S O L}$. The dashed lines denote a factor of 2 deviation from the regression.

the average Mach number $M=0.033,0.1$ and 0.3 was performed. The variation in $M$ leads to a change of the parallel loss time to the divertor $\tau_{S O L \rightarrow \text { div }}$ according to eq. 5 and 6. It varies across the divertor SOL and during time due to the dependence on the temperature (see eq.6). The spatially and temporally averaged parallel loss time from SOL to divertor $\left\langle\left\langle\tau_{S O L \rightarrow \text { div }}\right\rangle\right\rangle$ was around $6.6,2.2$ and $0.72 \mathrm{~ms}$ for the three Mach numbers. The transport model for each input parameter set was solved until temporal quasi-equilibrium of all impurities was reached. Finally, the scan was repeated using the background profiles $n_{e}, T_{e}$ and $T_{i}$ of all three discharges. For each setting, the particle confinement time of tungsten

$$
\tau_{p}=\frac{\left\langle N_{W}\right\rangle}{\left\langle\Phi_{W}\right\rangle}
$$

was calculated from the average number of tungsten ions in the confined plasma $\left\langle N_{W}\right\rangle$ and the neutral production rate at the limiters $\left\langle\Phi_{W}\right\rangle .\left\langle\Phi_{W}\right\rangle$ also includes the atoms which promptly redeposit. A regression of $\tau_{p}$ on $f_{E L M}$, the spatially and temporally averaged parallel loss time from SOL to divertor $\left\langle\left\langle\tau_{S O L \rightarrow d i v}\right\rangle\right\rangle$ and $D_{S O L}$ describes all values within a factor of 2 as is shown in Fig.6. It yields

$$
\tau_{p}=1.4 \times 10^{-2} \mathrm{~s} \quad f_{E L M}^{-1.1}\left\langle\left\langle\tau_{S O L \rightarrow d i v}\right\rangle\right\rangle^{1.1} D_{S O L}^{0.1}
$$

for $\left[f_{E L M}\right]=\mathrm{s}^{-1},\left[\left\langle\left\langle\tau_{S O L \rightarrow \text { div }}\right\rangle\right\rangle\right]=\mathrm{s}$ and $\left[D_{S O L}\right]=\mathrm{m}^{2} \mathrm{~s}^{-1}$. The dependence on $D_{a n}^{E T B}$ was negligible, since this parameter was only scanned in a range, where the neo-classical diffusion coefficient is dominant. Thus, $\tau_{p}$ varies almost linearly with $1 / f_{E L M}$ and $\left\langle\left\langle\tau_{S O L \rightarrow \text { div }}\right\rangle\right\rangle$, while the dependence on $D_{S O L}$ is very weak. Qualitatively, the weak dependence on $D_{S O L}$ is due to the twofold effect of SOL diffusion, driving a radial flux from the source location to the inside as well as to the outside. Quantitatively, it is already known from the much simpler case with temporally and spatially constant $D_{S O L}$, 
$\tau_{S O L \rightarrow \text { div }}$ and a constant source at fixed radial distance $\Delta r=r-r_{\text {sep }}$ to the separatrix. With such fixed SOL parameters, analytical solutions of the transport equation can be found $[26,16]$ which yield a maximum of $\tau_{p}$ at $D_{S O L}^{\max } \approx \Delta r^{2} / \tau_{S O L \rightarrow d i v}$. Using this result and replacing $\Delta r$ and $\tau_{S O L \rightarrow d i v}$ by average values, the investigated data set delivers $D_{S O L}^{\max }$ around $0.5,1.7$ and $5 \mathrm{~m}^{2} \mathrm{~s}^{-1}$ for the three settings of $\left\langle\left\langle\tau_{S O L \rightarrow \text { div }}\right\rangle\right\rangle$. For the individual subsets with one fixed value of $\left\langle\left\langle\tau_{S O L \rightarrow \text { div }}\right\rangle\right\rangle$, one finds a dependence of $\tau_{p}$ on $D_{S O L}$ which is consistent with the analytical solution, i.e. $\tau_{p}$ rises when $D_{S O L}$ approaches $D_{S O L}^{\max }$. In the complete data set, this leads to a very weak dependence on the diffusion coefficient in the SOL. Thus, when knowing an experimental value for $\tau_{p}$ at a given ELM frequency $f_{E L M}$, the most robust modelling output is the parallel loss time in the SOL provided that the radial transport during the ELM is well described. However, lower values of $D_{S O L}$ produce more strongly peaked profiles near the source radius. The density profile near the source radius has a characteristic radial decay length

$$
\lambda_{S O L, d i v}=\sqrt{D_{S O L} \tau_{S O L \rightarrow \text { div }}}
$$

and high impurity concentrations appear at the source location for the lower values of $D_{S O L}$. In the limiter shadow, the characteristic decay length $\lambda_{S O L, \text { lim }}=\sqrt{D_{S O L} \tau_{S O L \rightarrow l i m}}$ also increases with increasing $D_{S O L}$.

\section{Application to the modelling of ASDEX Upgrade H-mode discharges}

The model was applied to three H-mode discharges, with $f_{E L M}=50,100$ and $200 \mathrm{~Hz}$, where the erosion rates and the concentration inside the pedestal top are known from measurement (see Tab.1). Due to the weak dependence of $\tau_{p}$ on $D_{S O L}$, the parallel losses to divertor and limiter were tuned to fit the measured tungsten concentrations $c_{W, 0.8}$ at $r / a \approx 0.8$ for three values of $D_{S O L}=0.1,0.32$ and $1 \mathrm{~m}^{2} \mathrm{~s}^{-1}$ and fixed $D_{a n}^{E T B}=0.032 \mathrm{~m}^{2} \mathrm{~s}^{-1}$. Carbon and oxygen influxes are set to a constant value and complete limiter recycling was assumed. The influx values were chosen to yield the measured concentrations $c_{C, 0.9}$ and $c_{O, 0.9}$ at $r / a=0.9$ (see Tab.1).

The neutral tungsten influx from divertor, inner column and low-field side limiters was measured by visible spectroscopy. We assume the influx from the outboard limiters to be the dominant source for the tungsten concentration in the confined plasma [4]. Thus, only $1 / 3$ of the low-Z impurity losses onto the limiters are considered for the $\mathrm{W}$ production where this choice reflects the measured ratio of $\mathrm{W}$ influx from the outboard limiters to the influx from the inner heat shield. Using this choice, the calculated confinement time of tungsten $\tau_{p, W}$ is an upper bound, since the effect of the heat shield source is neglected. The ELM fraction of the total $\mathrm{W}$ source is known to be around $70 \%$ (see section 2). Thus, the modelled $\mathrm{W}$ sputtering rate shall deliver the measured rates and ELM fraction. There is a very strong dependence of the sputtering yield on temperature in the $10 \mathrm{eV}$ region. However, measurements of $T_{i}$ and $T_{e}$ at the limiters are either not existing or far too uncertain, such that experimental values could not be used as input to the code. Thus, the maximum value during the ELM $T_{e, l i m}^{E L M}$ and 


\begin{tabular}{|c|c|c|c|c|c|c|c|c|c|}
\hline & \multicolumn{3}{|c|}{ \# 22898} & \multicolumn{3}{|c|}{ \# 22895} & \multicolumn{3}{|c|}{ \# 22901} \\
\hline$\Phi_{D, p u f f}\left[10^{22} \mathrm{~s}^{-} 1\right]$ & \multicolumn{3}{|c|}{1.5} & \multicolumn{3}{|c|}{1} & \multicolumn{3}{|c|}{0.1} \\
\hline$f_{E L M}[\mathrm{~Hz}]$ & \multicolumn{3}{|c|}{200} & \multicolumn{3}{|c|}{100} & \multicolumn{3}{|c|}{50} \\
\hline$\left\langle\Phi_{W, \text { lim }}\right\rangle\left[10^{18} \mathrm{~s}^{-} 1\right]$ & \multicolumn{3}{|c|}{2.0} & \multicolumn{3}{|c|}{3.3} & \multicolumn{3}{|c|}{3.6} \\
\hline$c_{W, \text { lim }}^{E L M}$ & \multicolumn{3}{|c|}{0.7} & \multicolumn{3}{|c|}{0.7} & \multicolumn{3}{|c|}{0.7} \\
\hline$\left\langle c_{W, 0.8}\right\rangle\left[10^{-5}\right]$ & \multicolumn{3}{|c|}{0.84} & \multicolumn{3}{|c|}{1.8} & \multicolumn{3}{|c|}{3.6} \\
\hline$\left\langle c_{C, 0.9}\right\rangle,\left\langle c_{O, 0.9}\right\rangle[\%]$ & \multicolumn{3}{|c|}{$0.9,0.23$} & \multicolumn{3}{|c|}{$1.2,0.3$} & \multicolumn{3}{|c|}{$2.1,0.51$} \\
\hline$D_{S O L}\left[\mathrm{~m}^{2} \mathrm{~s}^{-1}\right]$ & 0.1 & 0.32 & 1 & 0.1 & 0.32 & 1 & 0.1 & 0.32 & 1 \\
\hline$T_{e, \lim }[\mathrm{eV}]$ & 7.7 & 7.1 & 6.7 & 11 & 9.5 & 8.3 & 12 & 10 & 8.2 \\
\hline$T_{e, l i m}^{E L M}[\mathrm{eV}]$ & 13 & 12 & 11 & 26 & 22 & 18 & 36 & 28 & 21 \\
\hline$\left\langle\left\langle\tau_{S O L \rightarrow \text { div }}\right\rangle\right\rangle[\mathrm{ms}]$ & 5.8 & 5.9 & 6.5 & 7.1 & 5.7 & 5.0 & 4.9 & 3.4 & 2.6 \\
\hline$\overline{\left\langle\left\langle\lambda_{S O L, d i v}\right\rangle\right\rangle[\mathrm{cm}]}$ & 4.5 & 6.1 & 9.2 & 3.9 & 5.1 & 7.5 & 2.9 & 3.7 & 5.3 \\
\hline$\left\langle\left\langle\lambda_{S O L, l i m}\right\rangle\right\rangle[\mathrm{mm}]$ & 2.8 & 4.3 & 6.9 & 2.8 & 4.1 & 6.1 & 2.4 & 3.3 & 4.8 \\
\hline$\left\langle n_{W, 0.9}\right\rangle /\left\langle n_{W, \text { sep }}\right\rangle$ & 4.4 & 3.9 & 3.6 & 8.9 & 8.1 & 7.8 & 22 & 21 & 20 \\
\hline$\left\langle n_{C, 0.9}\right\rangle /\left\langle n_{C, s e p}\right\rangle$ & 1.9 & 1.7 & 1.6 & 2.6 & 2.4 & 2.3 & 4.0 & 3.8 & 3.7 \\
\hline$\tau_{p, W}[\mathrm{~ms}]$ & \multicolumn{3}{|c|}{4.0} & \multicolumn{3}{|c|}{5.1} & \multicolumn{3}{|c|}{9.4} \\
\hline $1-\left\langle f_{\text {redep }}\right\rangle$ & 0.34 & 0.38 & 0.40 & 0.29 & 0.33 & 0.36 & 0.36 & 0.38 & 0.41 \\
\hline$\Delta N_{W} /\left\langle N_{W}\right\rangle[\%]$ & 6.7 & 8.4 & 9.7 & 7.9 & 9.7 & 10.6 & 8.7 & 9.7 & 10.3 \\
\hline
\end{tabular}

Table 1. Experimental and impurity transport modelling parameters for $\mathrm{W}$ edge transport of three H-mode discharges in ASDEX Upgrade. The first block gives experimental values. The second block comprises input parameters to the code or derived quantities, which are a direct consequence of the input parameters. The third block shows output values of the code, i.e the result of the impurity transport calculation.

the inter-ELM value of $T_{e, l i m}$ need to be prescribed in order to obtain the measured sputtering rates from the model. $T_{i, l i m}$ was set to $2 T_{e, l i m}$. The discharge with lowest deuterium puff and largest $\mathrm{W}$ influx, has the highest limiter temperatures. For each discharge, there is a small temperature variation with $D_{S O L}$. For increasing $D_{S O L}$, the radial decay length in the limiter shadow $\left\langle\left\langle\lambda_{S O L, \text { lim }}\right\rangle\right\rangle$ increases leading to a larger loss onto the limiters $\Phi_{\|, \text {lim }}$ (eq.8) and thus causing an increased tungsten sputtering rate. Therefore, the limiter temperatures, which are input to the model, need to be reduced with increasing $D_{S O L}$ to arrive at the measured tungsten influx as can be seen in Tab.1.

Finally, the Mach number is adjusted to fit the measured tungsten concentration $c_{W, 0.8}$. For the discharge \#22901, which has the lowest ELM frequency, the variation of the inter-ELM diffusion coefficient in the SOL $D_{S O L}$ has the strongest influence on $\left\langle D_{S O L}\right\rangle$ being the temporal average of the SOL diffusion coefficient during the ELM cycle. Here, the increase of $D_{S O L}$ by a factor of 10 needs the largest change in $\left\langle\left\langle\tau_{S O L \rightarrow \text { div }}\right\rangle\right\rangle$ and can be compensated by a factor of 2 decrease of $\left\langle\left\langle\tau_{S O L \rightarrow \text { div }}\right\rangle\right\rangle$ to get the same particle confinement time (the $\mathrm{W}$ confinement would increase with increasing $D_{S O L}$ at constant $\left.\left\langle\left\langle\tau_{S O L \rightarrow \text { div }}\right\rangle\right\rangle\right)$. The $\left\langle n_{W}\right\rangle$ profiles for the three $D_{S O L}$ cases are shown in 


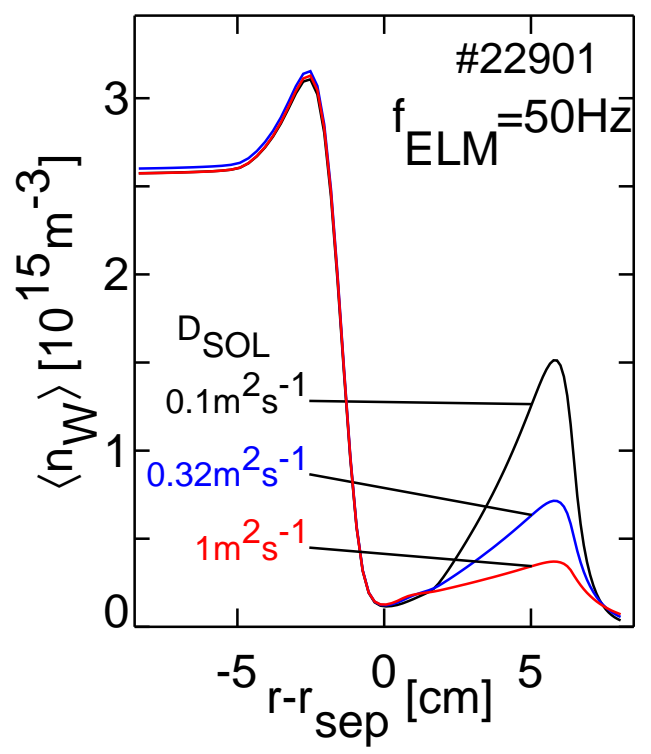

Figure 7. Modelled radial profiles of $\left\langle n_{W}\right\rangle$ yielding the measured $\mathrm{W}$ concentration at $r / a=0.8$ for the measured $\mathrm{W}$ influx for three different levels of the inter-ELM diffusion coefficient in the SOL.

Fig. 7 for \#22901. The profile for $D_{S O L}=0.1 \mathrm{~m}^{2} \mathrm{~s}^{-1}$ has a pronounced maximum around the source radius, and also for $\mathrm{C}$ and $\mathrm{O}$ very large concentrations around the source are found which might even lead to violations of the quasi-neutrality shortly after the ELM. Therefore, such low $D_{S O L}$ values are less probable. Furthermore, measurements of the $n_{W}$ decay lengths in the limiter shadow [27] of $\approx 5 \mathrm{~mm}$ also point in the direction of larger diffusion coefficients.

Besides the SOL transport issues, a strong effect of the ELM frequency on the average impurity density gradient is found. For decreasing ELM frequency, the ratio of the $\mathrm{W}$ density at $r / a=0.9$ to the separatrix value $\left\langle n_{W, 0.9}\right\rangle /\left\langle n_{W, s e p}\right\rangle$ increases somewhat stronger than linear, rising from 3.9 to 21 with a weak dependence on $D_{S O L}$. The dependence on $f_{E L M}$ simply reflects that the more frequent the evolution towards a strongly peaked profile in the ETB is reset by an ELM the lower is the average peaking. The weak dependence on $D_{S O L}$ can be understood when observing the flushing of tungsten from the confined region during an ELM. Each ELM reduces the tungsten content by $\Delta N_{W} /\left\langle N_{W}\right\rangle=6.7-11 \%$. For fixed ELM frequency, this flushing of tungsten becomes strongest when the density inside the pedestal top and in the SOL differs most, i.e. for the cases with $D_{S O L}=1 \mathrm{~m}^{2} \mathrm{~s}^{-1}$, where the tungsten density in the SOL is lowest (see Fig.7).

In general, the suppression of the neo-classical W peaking due to ELMs depends on the strength and the duration of the increased diffusion in the model and details can only be tested by impurity density measurements around the ETB with good spatial and temporal resolution. Previous application of the ELM model agreed well with $\mathrm{Si}$ and Ne measurements [22], however, fast measurements of $\mathrm{W}$ concentrations around the 
ETB are very difficult and still evolving [11]. In the present modelling approach, all ELMs are identical in terms of transport coefficients.

Finally, the prompt redeposition reduces the calculated tungsten confinement by a factor of 2.5-3 for these discharges with $B_{T}=2.5 \mathrm{~T}$. This can be seen from the time averaged fraction of $\mathrm{W}$ atoms, which do not promptly redeposit $\left(1-\left\langle f_{\text {redep }}\right\rangle\right.$ in Tab.1).

\section{Impurity transport in the ITER H-mode edge transport barrier}

The focus in this section is mainly on the perpendicular transport in the ETB and the consequences of a dominantly neo-classical impurity transport in the ETB between the ELMs. An attempt is made to calculate the average peaking of tungsten in the ETB of ITER plasmas for different ELM frequencies. It is an estimate for an ITER with tungsten PFCs in the main chamber, which might become relevant at a later stage of the ITER operation.

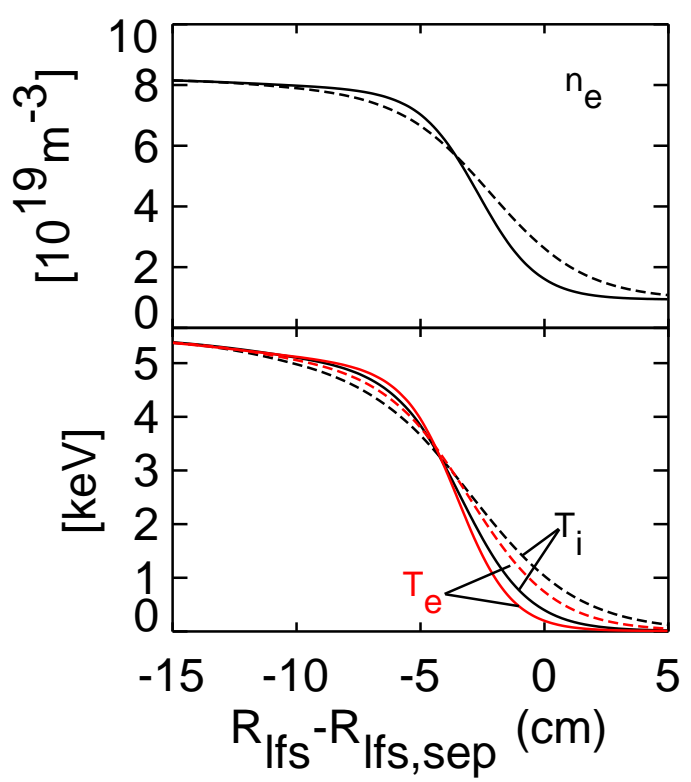

Figure 8. Edge profiles of $n_{e}, T_{e}$, and $T_{i}$ before (solid lines) and after a 1 MJ-ELM (dashed lines) used for the impurity transport calculation.

The calculations are for the ITER-FEAT reference scenario for inductive operation. The main parameters are: $R_{g e o}=6.2 \mathrm{~m}, a=2.4 \mathrm{~m}, B_{T}=5.3 \mathrm{~T}, I_{p}=15 \mathrm{MA}, P_{\mathrm{NBI}}=40 \mathrm{MW}$, $P_{\text {fus }}=400 \mathrm{MW}$, i.e $Q=10$. The central profiles up to the pedestal top are taken from transport calculations $[28,29]$ using heat and particle fluxes from the GLF23 code [30], which is based on ITG/TEM physics. The pedestal top values, that fulfil the $Q=10$ requirement are $T_{e, p e d}=T_{i, p e d}=4.8 \mathrm{keV}$, and $n_{e, p e d}=7.8 \times 10^{19} \mathrm{~m}^{-3}$. Modified tanhfunctions were used to describe the edge profiles at the low-field side equator

$$
y\left(R_{l f s}\right)=\frac{y_{p e d}+y_{S O L}}{2}+\frac{y_{p e d}-y_{S O L}}{2} \operatorname{mtanh}\left[-\frac{2 \lambda y_{p e d}^{\prime}}{y_{p e d}-y_{S O L}}, \frac{R_{c}-R_{l f s}}{\lambda}\right]
$$




$$
\operatorname{mtanh}[\alpha, z]=\frac{(1+\alpha z) \exp [z]-\exp [-z]}{\exp [z]+\exp [-z]}
$$

where $y_{p e d}$ is the pedestal top value, $y_{S O L}$ the SOL offset, $\lambda$ the decay length, $y_{\text {ped }}^{\prime}$ the asymptotic slope inside of the pedestal top, and $R_{c}$ the location of the pedestal centre [31]. For the pedestal width of $T_{e}$, a scaling with machine size was assumed and $\lambda=3.5 \times 10^{-3} R_{\text {geo }}$ was used [32]. The separatrix value of $T_{e}$ is quite well defined by parallel heat conduction [33]. It was set to $T_{e, s e p}=195 \mathrm{eV}$ and defines the central position of the function. The asymptotic slope inside the pedestal was set to $d T_{e, p e d} / d R=-5 \mathrm{keVm}^{-1}$ yielding a smooth transition to the core profile. The modified tanh-function without offset value in the SOL leads to very low electron temperatures at the limiters. Therefore, $T_{e, S O L}$ was set to $8 \mathrm{eV}$ to have some $\mathrm{W}$ erosion at the limiters due to physical sputtering by impurities. This value of $T_{e, S O L}$ is arbitrary and not important for the conclusions of this section. For the ion temperatures all parameters are the same, but the decay length was increased by $25 \%$ and $T_{i, s e p}=2 T_{e, s e p}$ was chosen, which defines the centre position of the $T_{i}$ function. The electron density decay in the ETB was calculated from the $T_{e}$ profile assuming $\eta_{e}=\left(d T_{e} / d R\right) /\left(d n_{e} / d R\right)=2[34,31]$ down to a an offset value of $1 \times 10^{19} \mathrm{~m}^{-3}$. The profiles after an ELM are constructed by increasing for all profiles the decay length by the same factor and shifting $R_{c}$ by the same amount, such that the total energy is conserved and that the ELM energy loss $\Delta W_{E L M}$ is shifted from the confined region into the SOL. This was done for the maximum permissible ELM energy loss in ITER, which is about $\Delta W_{E L M}=1 \mathrm{MJ}$ [35]. The profiles before and after an ELM are shown in Fig.8. Many of the assumptions are certainly questionable, however, for the further considerations only the high ETB temperatures and the scaling of the pedestal width with machine size are essential.

Fig.9 shows the calculated neo-classical transport coefficients between ELMs and the assumed anomalous profiles of the diffusion coefficient $D$ and of the drift parameter $v / D$. Additional impurities which contribute to the collisional transport of tungsten are helium with a concentration of $c_{\mathrm{He}, 0.9}=2 \%$, oxygen with $c_{0,0.9}=0.9 \%$, and Ar with $c_{\mathrm{Ar}, 0.9}=0.05 \%$. The concentration are chosen to yield a $Z_{\text {eff }}$ of $\approx 1.6$. corresponding to a dilution that is compatible with a fusion power of $400 \mathrm{MW}$. The neo-classical diffusion coefficient $D_{\text {neo }}$ in the ETB is shown for each impurity. For tungsten and argon, $D_{\text {neo }}$ is strongly dominated by the PS contribution, while for He the banana-plateau term $D_{B P}$ contributes about $60 \%$ at $r-r_{s e p}=-20 \mathrm{~cm}$ and only $10 \%$ near the separatrix. In the radial range, where $D_{B P}$ is important, the approximate banana width $w \approx \sqrt{\epsilon} \rho_{p}$ of all ions are well below the gradient lengths of temperature and density and the basic assumption of neo-classical transport theory is valid. The values of $D_{\text {neo }}$ are in the range $0.01-0.03 \mathrm{~m}^{2} \mathrm{~s}^{-1}$ and thus more than an order of magnitude below the typical values in ASDEX Upgrade. In order to have a dominant neo-classical impurity transport in the ETB the anomalous diffusion coefficient was reduced from $1 \mathrm{~m}^{2} \mathrm{~s}^{-1}$ at the pedestal top to an order of magnitude smaller values than $D_{\text {neo }}$, i.e. $1 \times 10^{-3} \mathrm{~m}^{2} \mathrm{~s}^{-1}$ in the ETB, and increased again to $0.5 \mathrm{~m}^{2} \mathrm{~s}^{-1}$ in the SOL in order to avoid very small characteristic length scales in the divertor SOL (see sec.5). The ELM is again induced by the same recipe as 


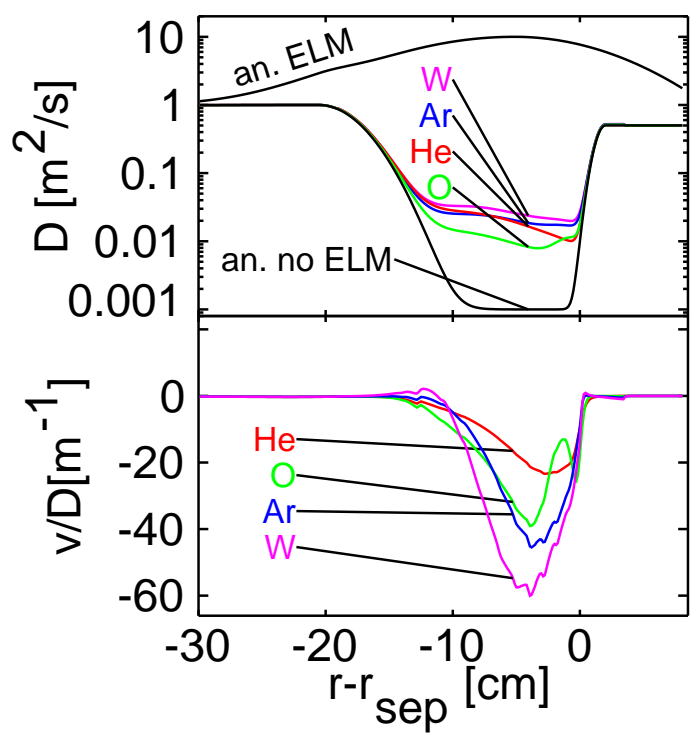

Figure 9. Radial profiles of the transport coefficients used in the ITER transport model. In the upper box, the lower solid black line (an. no ELM) is the assumed turbulent diffusion coefficient between ELMs $D_{a n}^{n o E L M}$, the coloured lines are the sum of neoclassical diffusion coefficients $D_{\text {neo }}$ and $D_{a n}^{n o} E L M$ for $\mathrm{He}, \mathrm{O}, \mathrm{Ar}$ and $\mathrm{W}$, and the upper solid black line (an. ELM) is the diffusion coefficient at the start of the ELM. In the lower box, the different lines represent $v_{\text {neo }} /\left(D_{\text {neo }}+D_{a n}^{n o E L M}\right)$ for all elements in the inter-ELM phase.

before (upper black curve), a sudden switch on of a large diffusion coefficient decaying linearly within $1 \mathrm{~ms}$.

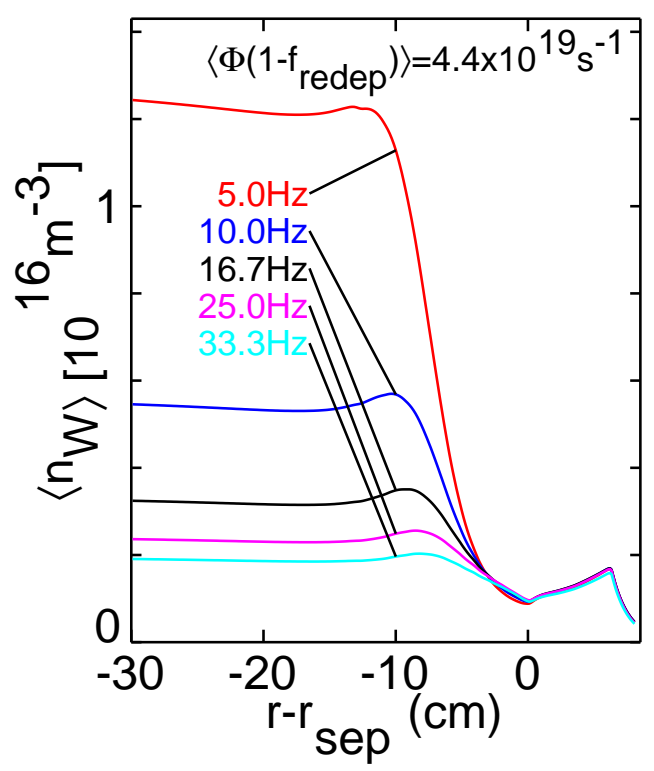

Figure 10. Modelled tungsten profiles in the ITER edge averaged over one ELM cycle. The profiles were calculated for different ELM frequencies and are normalised to the same effective source rate $\left\langle\Phi\left(1-f_{\text {redep }}\right)\right\rangle$. 
A rough estimate of the ELM frequency can be gained from the assumption that $1 / 3$ of the power transported across the separatrix $P_{\text {sep }}$ is exhausted within ELMs [36]. Using $P_{\text {heat }}=120 \mathrm{MW}$ and $P_{\text {rad,core }}=0.25 P_{\text {heat }}$ gives $P_{\text {sep }}=90 \mathrm{MW}$ and $f_{E L M}=30 \mathrm{~Hz}$. A scan of the ELM frequency was performed using $f_{E L M}=33 \mathrm{~Hz}$ as the highest value and going down to $f_{E L M}=5 \mathrm{~Hz}$. For the case with highest ELM frequency, the influxes of oxygen and argon were set to have core concentrations $c_{0,0.9}=0.9 \%$ and $c_{\mathrm{Ar}, 0.9}=0.05 \%$. For Helium we considered the source due to fusion in the confined plasma, $100 \%$ recycling at the limiters and divertor recycling, which was calculated with a simple divertor chamber model which describes the pumping and recycling with decay time constants [37]. The parameters of the decay times were set to get a global confinement time of He

$$
\tau_{H e}^{*}=\frac{\left\langle N_{H e}\right\rangle}{\Phi_{\mathrm{He}, \text { fus }}}
$$

of about 5 times the energy confinement time $\tau_{E}$. For the other ELM frequencies the oxygen and argon influxes and the recycling model for He were left unchanged. Fig. 10 shows the modelled $\left\langle n_{W}\right\rangle$ profiles at the edge for the different ELM frequencies. All profiles are normalised to the same tungsten source rate. The absolute $\mathrm{W}$ densities can not be predicted and we just concentrate on the peaking in the ETB vicinity. The peaking is very low at the higher frequencies and there is only a remarkable increase when decreasing $f_{E L M}$ to $10 \mathrm{~Hz}$. At $f_{E L M}=10 \mathrm{~Hz}$ the tungsten peaking across the pedestal is $\left\langle n_{W, 0.9}\right\rangle /\left\langle n_{W, \text { sep }}\right\rangle \approx 6$ and at $f_{E L M}=5 \mathrm{~Hz}$ the peaking factor is about 14 . These values are in the range of peaking factors calculated for the ASDEX Upgrade ETB at about 20 times higher ELM frequencies (see Tab.1 at $f_{E L M}=100$ and $200 \mathrm{~Hz}$ ). For argon and oxygen, the peaking is even weaker. Thus, the neo-classical impurity peaking in the ETB seems to be of much lower importance in ITER due to the low neo-classical transport coefficients leading to equilibration times much longer than the foreseen ELM repetition time, where it is assumed that the small ELMs in ITER are effectively weakening the impurity gradient in the ETB. For the case with $f_{E L M}=5 \mathrm{~Hz}$, a series of runs were performed, where the turbulent diffusion coefficient in the ETB $D_{a n, E T B}$ was increased from the value of $0.001 \mathrm{~m}^{2} \mathrm{~s}^{-1}$ up to $0.1 \mathrm{~m}^{2} \mathrm{~s}^{-1}$. The peaking $\left\langle n_{W, 0.9}\right\rangle /\left\langle n_{W, s e p}\right\rangle$ steadily decreases with rising $D_{a n, E T B}$ and is reduced to half of the starting value when reaching $D_{a n, E T B}=0.014 \mathrm{~m}^{2} \mathrm{~s}^{-1}$, which approximately equals the collisional diffusion coefficient.

There remains the concern about the He exhaust, since He, which is created by fusion in the core of plasma, has to diffuse through the low transport region at the ETB. Four sets of calculations have been performed. For all sets, Fig.11 shows the global helium confinement time $\tau_{H e}^{*}$ and the ratio with $\tau_{E} \approx 2.5 \mathrm{~s}$ as a function of ELM frequency. In the first set (full symbols in Fig.11), the helium which is transported onto the limiters is re-injected in the next time step, i.e. with complete limiter recycling, while He ions that are lost to the divertor do not return. This yields a very low total He recycling source on the order of the fusion source and represents the case which is dominated by the perpendicular transport across the ETB. For this set $\tau_{H e}^{*}$ increases from $4.2 \mathrm{~s}$ at $f_{E L M}=33 \mathrm{~Hz}$ to $12 \mathrm{~s}$ at $f_{E L M}=5 \mathrm{~Hz}$. When including the simple divertor recycling model [37], the source in the SOL is about 100 times stronger than the fusion 


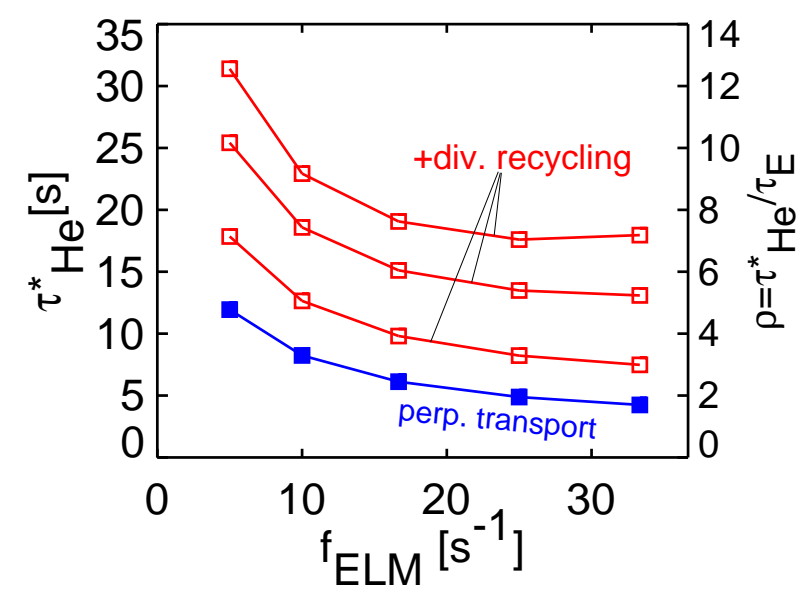

Figure 11. Effect of the ELM frequency $f_{E L M}$ on the global helium confinement time $\tau_{H e}^{*}$ in ITER. The blue curve is for the case without He recycling from the divertor, where the perpendicular transport through the ETB is the only limiting factor. The red curves are for three assumed levels of divertor recycling which cause a further increase of $\tau_{H e}^{*}$.

source. The value of $\tau_{H e}^{*}$ at $f_{E L M}=33 \mathrm{~Hz}$ is determined by the time constants in the model and was set to three different values: $7.5 \mathrm{~s}, 13 \mathrm{~s}$, and $18 \mathrm{~s}$. The other values of the scans are calculated without changing the respective time constants. For these scans, the effect of the changing perpendicular transport with decreasing ELM frequency is less visible for the cases with large divertor recycling. In all cases a strong increase of $\tau_{H e}^{*}$ is observed for $f_{E L M}=5 \mathrm{~Hz}$, where $\tau_{H e}^{*}$ is $17.8 \mathrm{~s}, 25.4 \mathrm{~s}$ and $31.4 \mathrm{~s}$ for the respective scans. The divertor recycling model certainly is a crude simplification for the fuelling from the divertor and shall just demonstrate that the perpendicular transport effects in the ETB are not very important as long as the ELM frequency is in the expected range around $20 \mathrm{~Hz}$ even for neo-classical diffusion in the ETB. The details of the change of $\tau_{H e}^{*}$ with ELM frequency depend of course on the value, the radial width and the duration of an increased diffusion coefficient during the ELM.

\section{Conclusions}

Fast measurements of the total erosion rates at the plasma facing components in ASDEX Upgrade have shown that at the outboard limiters $70 \pm 10 \%$ of the erosion is due to ELMs, while at the inboard heat shield the contribution is lower at a level of $40 \pm 10 \%$. The ELM contribution does not depend on the energy loss during an ELM, i.e. less frequent larger ELMs cause the same contribution as more frequent smaller ELMs. The ratio of outboard to inboard erosion rates decreases when the plasma is shifted to the inboard. However, the balance is different during an ELM and between ELMs with a stronger outboard contribution during an ELM.

For tungsten, the transport in the ETB is dominated by the effect of Coulomb 
collisions, since it has an even higher collisional diffusion coefficient than the light elements, for which the agreement with purely collisional transport could be deduced from direct measurements. The collisional transport produces a strong inward pinch and a respective peaking of tungsten in the ETB which is relaxed during an ELM. When modelling the edge transport in ASDEX Upgrade with a 1D radial transport code that implements the parallel losses in the SOL as volume loss rates, the impurity confinement time shows a strong decrease with increasing ELM frequency as experimentally observed. However, there is an equally strong dependence on the characteristic parallel loss time in the SOL, which is at present not well known and requires further measurements to restrict this parameter in the model. The dependence on the diffusion coefficient in the SOL is very weak and can hardly be determined from measured confinement times.

In the ETB of ITER, the collisional impurity diffusion coefficient is about a factor of 20 smaller than in ASDEX Upgrade. Thus the time to build up a strong edge gradient is substantially longer, however, the ELM frequency has to be kept high (around 20$30 \mathrm{~Hz}$ ) in order to achieve small ELMs. Thus, the average edge peaking will be quite small, provided that the ELMs do not lead to a much smaller increase of the effective diffusion coefficient than used in the model. Then, the high ELM frequency also helps to transport the helium across the plasma edge and does not lead to an unacceptably large value of $\tau_{H e}^{*}$.

\section{References}

[1] H. Bolt, V. Barabash, W. Krauss, J. Linke, R. Neu, et al., Materials for the Plasma-facing Components of Fusion Reactors, Journal of Nuclear Materials 329-333, 66-73 (2004).

[2] T. Pütterich, R. Neu, R. Dux, A. Whiteford, M. O'Mullane, et al., Calculation and experimental test of the cooling factor of tungsten, Nuclear Fusion 50(2), 025012 (9pp) (2010).

[3] R. Dux, R. Neu, C. F. Maggi, A. G. Petters, R. Pütterich, et al., Impurity Transport and Control in ASDEX Upgrade, in Fusion Energy 2004 (Proc. 20th Int. Conf. Vilamoura, 2004), (Vienna: IAEA) CD-ROM file EX/P6-14 and http://wwwnaweb.iaea.org/napc/physics/fec/fec2004/datasets/index.html

[4] R. Dux, V. Bobkov, A. Herrmann, A. Janzer, A. Kallenbach, et al., Plasma-wall Interaction and Plasma Behaviour in the Non-boronised all Tungsten ASDEX Upgrade, Journal of Nuclear Materials 390-391, 858-863 (2009).

[5] K. Krieger, T. Lunt, R. Dux, A. Janzer, A. Kallenbach, et al., Induced tungsten melting events in the divertor of ASDEX Upgrade and their influence on plasma performance, Journal of Nuclear Materials at press, doi: $10.1016 / \mathrm{j}$.jnucmat.2010.12.229.

[6] T. Lunt, Y. Feng, K. Krieger, A. Kallenbach, R. Neu, et al., 3D modeling of the ASDEX Upgrade edge plasma exposed to a localized tungsten source by means of EMC3-Eirene, Journal of Nuclear Materials at press, doi: 10.1016/j.jnucmat.2010.11.009.

[7] A. Geier, H. Maier, R. Neu, K. Krieger, and ASDEX Upgrade Team, Determination of the Tungsten Divertor Retention at ASDEX Upgrade using a Sublimation Probe, Plasma Physics and Controlled Fusion 44(10), 2091-2100 (2002).

[8] T. Pütterich et al., ELM Flushing and Impurity Transport in the H-Mode Edge Barrier in ASDEX Upgrade, Journal of Nuclear Materials at press, doi: 10.1016/j.jnucmat.2010.09.052.

[9] T. Pütterich, R. Dux, E. Wolfrum, E. Viezzer, and ASDEX Upgrade Team, Impurity Transport within an ELM-cycle at the Edge Transport Barrier in ASDEX Upgrade, in Europhysics Conference Abstracts (CD-ROM, Proc. of the 36th EPS Conference on Plasma Physics, Sofia, 
Bulgaria, 2009), edited by M. Mateev and E. Benova, volume 33E of ECA, pages $\mathrm{P}-1.158$, Geneva, 2009, European Physical Society.

[10] T. Pütterich, R. Neu, R. Dux, A. D. Whiteford, M. G. O’Mullane, et al., Modelling of Measured Tungsten Spectra from ASDEX Upgrade and Predictions for ITER, Plasma Physics and Controlled Fusion 50(8), 085016 (2008).

[11] A. Janzer, R. Dux, T. Pütterich, and ASDEX Upgrade Team, Investigating Tungsten Transport in the Plasma Edge at ASDEX Upgrade, in Europhysics Conference Abstracts (CD-ROM, Proc. of the 37th EPS Conference on Plasma Physics, Dublin, Ireland, 2010), edited by C. McKenna, volume 34A of ECA, page P1.1046, Geneva, 2010, European Physical Society and http://ocs.ciemat.es/EPS2010PAP/pdf/P1.1046.pdf

[12] R. Dux, V. Bobkov, N. Fedorczak, K. Iraschko, A. Kallenbach, et al., Tungsten Erosion at the ICRH Limiters in ASDEX Upgrade, Journal of Nuclear Materials 363-365, 112-116 (2007).

[13] J. Steinbrink, U. Wenzel, W. Bohmeyer, G. Fußmann, and P. Team, Sputtered Tungsten Atoms Investigated in a Linear Plasma Generator, in Europhysics Conference Abstracts (Proc. of the 24th EPS Conference on Controlled Fusion and Plasma Physics, Berchtesgaden, 1997), edited by M. Schittenhelm, R. Bartiromo, and F. Wagner, volume 21A, part IV, pages 1809-1812, Petit-Lancy, 1997, EPS.

[14] A. Thoma, K. Asmussen, R. Dux, K. Krieger, A. Herrmann, et al., Spectroscopic Measurements of Tungsten Erosion in the ASDEX Upgrade Divertor, Plasma Physics and Controlled Fusion 39(9), 1487-1499 (1997).

[15] R. Dux, STRAHL Manual, Rep. IPP 10/30, Max-Planck-Institut für Plasmaphysik, Garching, 2006.

[16] R. Dux, Impurity Transport in Tokamak Plasmas, Rep. IPP 10/27, Max-Planck-Institut für Plasmaphysik, Garching, 2004.

[17] PEETERS, A. G., Reduced charge state equations that describe Pfirsch Schlüter impurity transport in tokamak plasma, Phys. of Plasmas 7 (2000) 268.

[18] P. Stangeby, The Plasma Boundary of Magnetic Fusion Devices, Institute of Physics Publishing, Bristol and Philadelphia, 2000.

[19] G. A. Emmert, R. M. Wieland, A. T. Mense, and J. N. Davidson, Electric sheath and presheath in a collisionless, finite ion temperature plasma, Phys. Fluids 23, 803-812 (1980).

[20] W. Eckstein, C. García-Rosales, and J. Roth, Threshold energy for sputtering and its dependence on angle of incidence, Nucl. Instrum. Methods Phys. Research B 83, 95-109 (1993).

[21] M. W. Thompson, The energy spectrum of ejected gold atoms during the high energy sputtering of gold, Phil. Mag. 18, 377-414 (1968).

[22] R. Dux, Impurity Transport in ASDEX Upgrade, Fusion Science and Technology 44(3), 708-715 (2003).

[23] R. Dux, R. Neu, A. G. Peeters, G. Pereverzev, A. Mück, et al., Influence of the Heating Profile on Impurity Transport in ASDEX Upgrade, Plasma Physics and Controlled Fusion 45(9), 1815-1825 (2003).

[24] M. Sertoli, C. Angioni, R. Dux, R. Neu, T. Pütterich, et al., Local effects of ECRH on argon transport in L-mode discharges at ASDEX Upgrade, Plasm. Phys. and Contr. Nucl. Fus. 55, 035024 (2011).

[25] R. Dux, T. Pütterich, A. Janzer, and ASDEX Upgrade Team, Flushing and Erosion of Tungsten during Edge Localised Modes, in Europhysics Conference Abstracts (CD-ROM, Proc. of the 36th EPS Conference on Plasma Physics, Sofia, Bulgaria, 2009), edited by M. Mateev and E. Benova, volume 33E of ECA, pages O-5.056, Geneva, 2009, European Physical Society and http://epsppd.epfl.ch/Sofia/pdf/O5_056.pdf

[26] D. E. Post and K. Lackner, Plasma Models for Impurity Control Experiements, in Physics of Plasma-Wall Interactions in Controlled Fusion Devices, edited by R. Behrisch and D. Post, pages 627-692, New York, 1986, Plenum Press.

[27] W. Schustereder, K. Krieger, A. Herrmann, V. Rohde, and ASDEX Upgrade Team, Discharge 
Resolved Impurity Flux Measurements in the Edge Plasma of ASDEX Upgrade by Exposure of Collector Probes, Journal of Nuclear Materials 363-365, 242-246 (2007).

[28] G. V. Pereverzev and O. V. Zolotukhin, Predictive Simulation of ITER Performance with Theory-Based Transport Models, in Europhysics Conference Abstracts (CD-ROM, Proc. of the 30th EPS Conference on Controlled Fusion and Plasma Physics, St. Petersburg, 2003), edited by R. Koch and S. Lebedev, volume 27A, pages P-3.138, Geneva, 2003, EPS and http://epsppd.epfl.ch/StPetersburg/pdf/P3_138.pdf

[29] G. N. Pereverzev, C. Angioni, A. G. Peeters, and O. V. Zolotukhin, Theoretical Predictions of the Density Profile in a Tokamak Reactor, Nuclear Fusion 45(4), 221-225 (2005).

[30] R. E. Waltz, G. M. Staebler, W. Dorland, G. W. Hammett, M. Kotschenreuther, et al., A gyroLandau-fluid transport model, Phys. Plasmas 4, 2482-2496 (1997).

[31] A. Kallenbach, R. Dux, J. Gafert, G. Haas, L. D. Horton, et al., Edge Transport and its Interconnection with Main Chamber Recycling in ASDEX Upgrade, Nuclear Fusion 43(7), $573-578(2003)$.

[32] A. Kallenbach, N. Asakura, A. Kirk, A. Korotkov, M. A. Mahdavi, et al., Multi-machine Comparisons of H-mode Separatrix Densities and Edge Profile Behaviour in the ITPA SOL and Divertor Physics Topical Group, Journal of Nuclear Materials 337-339, 381-385 (2005).

[33] A. Kallenbach, M. N. A. Beurskens, A. Korotkov, P. Lomas, W. Suttrop, et al., Scaling of the Pedestal Density in Type-I ELMy H-mode Discharges and the Impact of Upper and Lower Triangularity in JET and ASDEX Upgrade, Nuclear Fusion 42(10), 1184-1192 (2002).

[34] J. Neuhauser, D. Coster, H. U. Fahrbach, J. C. Fuchs, G. Haas, et al., Transport into and across the Scrape-off Layer in the ASDEX Upgrade Divertor Tokamak, Plasma Physics and Controlled Fusion 44(6), 855-869 (2002).

[35] W. Fundamenski, Power and Particle Exhaust in Tokamaks: Integration of Plasma Scenarios with Plasma Facing Materials and Components, J. Nucl. Mater. 390-391, 10-19 (2009).

[36] T. Eich, P. Andrew, A. Herrmann, W. Fundamenski, A. Loarte, et al., ELM Resolved Energy Distribution Studies in JET MKII Gas-Box Divertor Using Infra-red Thermography, Plasma Physics and Controlled Fusion 49(5), 573-604 (2007).

[37] A. Kallenbach, R. Dux, V. Mertens, O. Gruber, G. Haas, et al., H-Mode Discharges with FeedbackControlled Radiative Boundary in the ASDEX Upgrade Tokamak, Nuclear Fusion 35(10), 1231-1246 (1995). 